\title{
छூ \\ Emittance conservation by tailored focusing profiles in a plasma accelerator
}

\author{
I. Dornmair, ${ }^{1,2}$ K. Floettmann, ${ }^{3}$ and A. R. Maier ${ }^{1,2, *}$ \\ ${ }^{1}$ CFEL, Center for Free-Electron Laser Science, 22607 Hamburg, Germany \\ ${ }^{2}$ University of Hamburg, Institute of Experimental Physics, 22761 Hamburg, Germany \\ ${ }^{3}$ DESY, 22607 Hamburg, Germany
}

(Received 10 July 2014; published 30 April 2015)

\begin{abstract}
Laser-plasma accelerators, providing high electric field gradients, are promising candidates to drive next-generation compact light sources and high-energy applications. However, conservation of beam emittance, a prerequisite for future applications, is very challenging, as the accelerated beam has to be matched to the plasma's strong focusing forces. Here we derive with simulations ideal laser and plasma density profiles to match an electron beam in and out of a plasma stage, thus relaxing required beta functions for injection and minimizing divergence and emittance growth after the plasma.
\end{abstract}

DOI: 10.1103/PhysRevSTAB.18.041302

PACS numbers: 29.27.-a, 41.75.Fr, 41.85.Ar, 52.38.Kd

\section{INTRODUCTION}

In a laser-plasma accelerator $[1,2]$ a driver laser pulse of relativistic intensity trails a plasma wakefield, generating strong radial and longitudinal electric fields. This acceleration cavity can be used either to accelerate electrons captured directly from the plasma background (selfinjection, triggered injection [2]) or to boost preaccelerated beams (external injection), which is especially relevant for a staged sequence of plasma accelerators currently being considered for high-energy physics $[3,4]$. Acceleration of self-injected electron beams to $\mathrm{GeV}$ energies over centimeter distances has been experimentally demonstrated [5-7]. However, the beams typically show divergences of several milliradians and broad energy spectra, caused by off-crest acceleration, phase slippage between the driver laser and accelerated electron beam, and from filling a broad range of the accelerating phase during injection.

Recently, it has been pointed out $[8,9]$ that beams featuring such properties are not suitable for further beam transport and applications, as the large divergence poses extreme requirements on the following beam optics and, in combination with the energy spread, causes a strong emittance growth in the drift after the plasma [10]. As discussed in Ref. [11], also single-shot measurements of the emittance after extraction from the plasma are challenging because of the small beam size in the plasma. Inside the plasma, extremely good emittances have already been measured [12], enabled by the strong focusing forces. It has been suggested that properly tapering the plasma density

\footnotetext{
"andreas.maier@desy.de
}

Published by the American Physical Society under the terms of the Creative Commons Attribution 3.0 License. Further distribution of this work must maintain attribution to the author(s) and the published article's title, journal citation, and DOI. at the exit may reduce the divergence $[8,9,13,14]$. Yet, this idea was not further elaborated.

What actually is desired is an adiabatic reduction of the focusing forces at the plasma-vacuum transition. Here, we show that only in special cases can this be achieved by a density taper, while in general also the driver laser propagation needs to be tailored.

Closely related to the extraction, an externally injected electron beam has to be focused to exceptionally small beta functions at the plasma entrance to match it to the strong focusing, requiring also strongly focusing injection beam optics, leading to long coupling sections between subsequent elements of a staged accelerator [15]. This strongly focusing injection optics before the plasma also amplifies beam jitter. In the plasma, a transverse mismatch of the external beam with the driver laser-and thus the plasma channel-induces a rapid emittance growth [16].

In this paper, we discuss the proper injection of an external electron beam into a plasma stage and the following extraction of a finite energy spread beam from the plasma with the help of adiabatic matching sections. We derive ideal laser and plasma density profiles that significantly increase the beta function and positioning jitter tolerances for injection and minimize the divergence and emittance growth of the extracted beam. Exemplarily, our method is illustrated with the simulation of a $100 \mathrm{MeV}$ beam that is injected into a $21 \mathrm{~cm}$ long plasma stage, accelerated to $1 \mathrm{GeV}$ and extracted without a loss of beam quality.

\section{IDEAL TAPERING PROFILES}

In the following, we consider an electron beam described by the Courant-Snyder parameters $\beta=\left\langle x^{2}\right\rangle / \epsilon$ and $\alpha=$ $-\left\langle x x^{\prime}\right\rangle / \epsilon$. Here, $\epsilon=\sqrt{\left\langle x^{2}\right\rangle\left\langle x^{\prime 2}\right\rangle-\left\langle x x^{\prime}\right\rangle^{2}}$ is the transverse rms emittance, $\beta$ is a measure of the transverse beam size in $x$, and $\alpha$ correlates the particle trajectory $x$ and its slope $x^{\prime}$. In the plasma, the driver laser creates a wakefield in the 
linear $\left(a=e A / m_{e} c^{2} \ll 1\right)$ regime [2,17] with longitudinal and radial electric fields

$$
\begin{gathered}
E_{z}(r, \zeta) \propto a^{2} k_{p}^{2} \exp \left(-\frac{k_{p}^{2} \sigma_{z}^{2}}{2}-\frac{2 r^{2}}{w^{2}}\right) \cos (\Psi), \\
E_{r}(r, \zeta) \propto-\frac{a^{2} k_{p} r}{w^{2}} \exp \left(-\frac{k_{p}^{2} \sigma_{z}^{2}}{2}-\frac{2 r^{2}}{w^{2}}\right) \sin (\Psi),
\end{gathered}
$$

with $\Psi=k_{p} \zeta$, the comoving variable $\zeta=z-v_{g} t$, the laser group velocity $v_{g}$, and the plasma frequency $k_{p} c=\left(n e^{2} / m_{e} \epsilon_{0}\right)^{1 / 2}$. We assume a Gaussian laser pulse of normalized vector potential $a^{2}=a_{0}^{2} \exp \left(-2 r^{2} / w^{2}\right)$ $\exp \left[-\zeta^{2} /\left(2 \sigma_{z}^{2}\right)\right]$ and length $\sigma_{z}$. Near the axis, $E_{r}$ causes a focusing strength $K$ of

$$
\begin{aligned}
K & =\left.\frac{e}{\gamma m_{e} c^{2}} \partial_{r} E_{r}\right|_{r=0} \\
& \propto-\frac{a^{2} k_{p}}{w^{2}} \exp \left(-k_{p}^{2} \sigma_{z}^{2} / 2\right) \sin (\Psi),
\end{aligned}
$$

and the beam is matched for $\beta=1 / \sqrt{K}$, with $\gamma$ the normalized electron beam energy. A matched beam has a constant beam envelope, unlike a mismatched beam, whose transverse phase space rotates with the betatron frequency $\omega_{\beta}$. The betatron frequency is a function of $K$ and consequently $\gamma$. If bunch electrons experience varying $K$ (due to a finite bunch length) or have different energies, the phase space ellipses of those electrons will rotate with different betatron frequencies, causing an increase in projected emittance. To prevent emittance growth, the whole beam has to be matched [15].

The focusing strength is proportional to the transverse electric field gradient and therefore depends on the phase $\Psi$ and the slowly varying local quantities $n$, the plasma density determining the peak electric field, and $w$, the laser spot size setting the wakefield width. To obtain a desired focusing profile, we have two free parameters: $w(z)$ and $n(z)$. A discharge capillary waveguide allows one to select both by designing a proper target geometry [18].

In general, a plasma accelerator stage consists of three sections: (i) an up ramp of the plasma density, (ii) a constant (or slightly tapered) density for acceleration, and (iii) a density down ramp to extract the accelerated beam.

\section{A. Injection}

The discussion of the plasma up ramp is relevant only for external injection, including staged accelerator schemes. We assume that the laser follows the paraxial laser evolution in the density up ramp, with the laser focus at the beginning of the acceleration section, and is then guided with a plasma channel until the end of the extraction section.

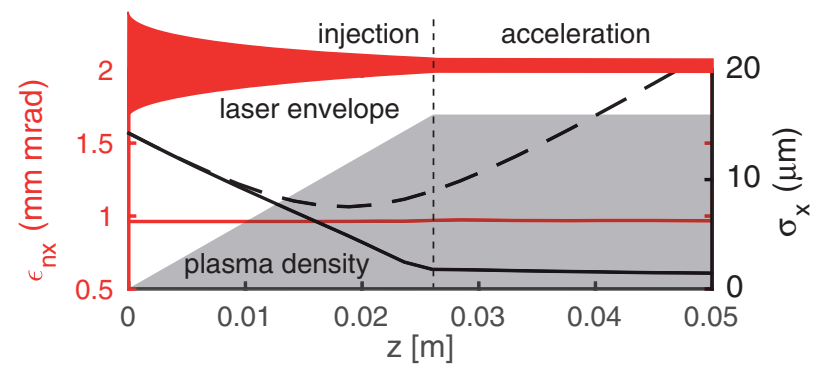

FIG. 1. Beam size (solid black line) and emittance evolution (red line) in the injection section. The dashed line represents the beam size evolution without the plasma target to illustrate the position and size of the virtual electron focus in the injection section.

At the beginning of the acceleration section, the electron beta function needs to be matched to the corresponding plasma density $n_{\text {accel }}$, and $\alpha=0$ is required. Typically, a plasma target with a sharp vacuum-plasma transition is considered, and the electron beam is focused at the start of the plasma to match it to the focusing forces [15]. This causes extreme sensitivity to emittance growth from transversely mismatched beams and demands a strong focusing injection optics.

By adding a smooth vacuum-plasma transition of length $l_{\text {inj }}$ before the acceleration section, an externally injected bunch already experiences focusing forces as it propagates through the density up ramp. Neglecting space charge, the beam envelope follows the differential envelope equation $\sigma_{x}^{\prime \prime}+K(z) \sigma_{x}-\epsilon^{2} \sigma_{x}^{-3}=0$. The additional focusing, similar to a thick lens, relaxes the injection beam optics. By backpropagating the matched beam through the density up ramp, we calculate the alpha and beta functions at the beginning of the up ramp and derive a "virtual focus" in the plasma to which we tune the injection beam optics (see Fig. 1). The virtual focus has a larger beta function $\beta_{\text {foc }}$ compared to a scheme with a sharp vacuum-plasma transition.

\section{B. Acceleration}

Next, we discuss the acceleration in the plasma. For efficient acceleration, the bunch needs to be placed near the on-crest acceleration phase $\Psi \sim-\pi$ at the beginning of the acceleration section. It then slowly slips with respect to the laser. After acceleration to $1.0 \mathrm{GeV}$, using our present parameter set, it is extracted through the plasma-vacuum transition.

During acceleration, the bunch accumulates a large correlated energy spread due to its finite bunch length compared to the plasma wavelength, combined with offcrest acceleration and phase slippage. However, the emittance, as experimentally demonstrated [12,14], remains constant, since the beam is always matched to the focusing forces. Minimizing the energy spread growth is highly desirable; however, this is beyond the scope of this paper. 


\section{Extraction}

Since the matched spot size in the plasma is very small, the divergence is large. For a sharp plasma-vacuum transition, the bunch will therefore strongly diverge in the drift section. First, this causes large chromatic emittance growth, and, second, it requires extremely strong beam optical elements to capture the beam $[8,9]$. The aim of the extraction section therefore is to provide a low divergence beam and to conserve emittance. We achieve this by adiabatically decreasing the focusing forces. Adiabaticity is a measure for the phase advance per change in focusing strength $K$. For adiabatic focusing, the electron bunch is always matched. For decreasing $K$, the spot size increases, and, since the emittance is conserved, the divergence is reduced. As discussed before, $K(z)$ is a combination of the laser profile $w(z)$ and the plasma density $n(z)$.

Previously, we showed a general solution of an adiabatic matching section as [19]

$$
K(z)=K_{0} /(1+g z)^{4},
$$

with $g$ the adiabaticity parameter, and $g \beta_{0} \ll 1$ for $\beta_{0}$ the beta function at the beginning of the matching section. This profile provides the best divergence reduction at minimum length. Here, we apply our general concept to the specific problem of a laser-plasma accelerator. For laser-driven plasma acceleration, laser guiding beyond the Rayleigh length $[5,18]$ is typically achieved in a hydrogen-filled capillary target. As in principle this allows one to tune $w(z)$ and $n(z)$ independently, one has two options to adiabatically reduce the focusing forces in the extraction section, namely, either increasing $w(z)$ at constant $n$ or decreasing $n(z)$ while keeping $w$ constant. We find that, for beam dynamical reasons, tailoring $w(z)$ is the more robust alternative.

If the density is constant, then $K \propto w^{-4}$, and the ideal $w(z)$ is, following Eq. (4),

$$
w(z)=w_{0}(1+g z) .
$$

This is the approach we show in Figs. 2 and 4 (discussed below). As long as adiabaticity is fulfilled, it is not important that $w(z)$ follows exactly this profile. Small deviations can be compensated with slightly longer extraction sections.

For constant $w$, the focusing strength scales with the plasma density as $K \propto-k_{p} \exp \left(-k_{p}^{2} \sigma_{z}^{2} / 2\right) \sin \left(k_{p} \zeta\right)$. If the bunch is at the dephasing point at the start of the extraction section, $K\left(k_{p}\right)$ decreases monotonically for monotonically decreasing $k_{p}$, and the ideal $n(z)$ profile can be obtained by simply solving Eq. (4) for $k_{p}(z)$. For a resonantly driven wake in the acceleration section $\left(\sigma_{z}=1 / k_{p \text {,accel }}\right)$, we find to good approximation

$$
n(z)=n_{\text {accel }}\left[1+c_{1} g z+c_{2} g^{2} z^{2}\right]^{-2},
$$

with $c_{1}=3.45$ and $c_{2}=1.59$.
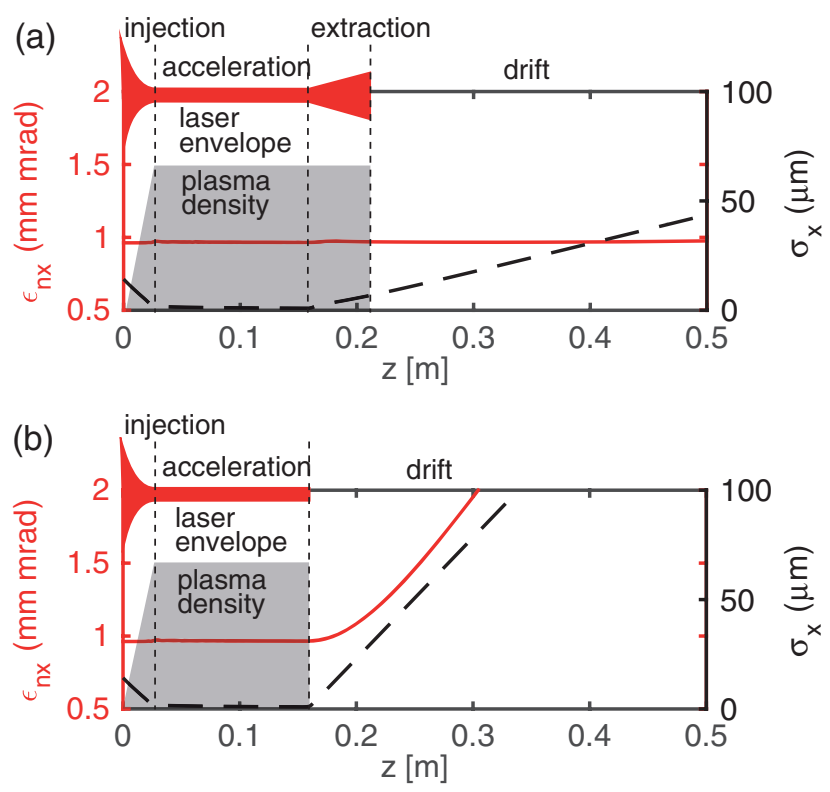

FIG. 2. Evolution of the electron beam size (black line) and emittance (red line) in the plasma booster stage. Adding an adiabatic extraction section (a), the beam emittance is effectively constant, $\Delta \epsilon_{n x} / \Delta z=0.18 \mathrm{~mm} \mathrm{mrad} / \mathrm{m}$, with a minimum beam divergence of only $130 \mu \mathrm{rad}$. In (b), the beam is extracted through the sharp ending of the focusing force, with a beam divergence of $550 \mu \mathrm{rad}$ and an emittance growth of $\Delta \epsilon_{n x} / \Delta z=12 \mathrm{~mm} \mathrm{mrad} / \mathrm{m}$, over one order of magnitude larger compared to the adiabatic extraction (a). Here, the gray areas indicate the plasma density profile, and the red areas schematically show the laser envelope. The laser evolution after the plasma is not shown.

\section{SIMULATIONS}

In this section, we demonstrate the applicability of our concept by employing numerical simulations. We show the increased tolerance to positioning jitters caused by a dedicated injection section as well as the divergence reduction while conserving the emittance in the extraction section. Especially for the jitter simulations, a large number of runs is needed to provide sufficient statistics. For this reason, we included the above-mentioned linear wakefield model, Eqs. (1) and (2), into the particle tracking code ASTRA [20], which is very fast compared to particle-in-cell (PIC) codes. Within certain limitations, the linear wakefield model in ASTRA provides a sufficient description of the physics involved. It includes phase shifts of the bunch caused both by slippage and by plasma density variations. Wakefield nonlinearities, pump depletion, and the effect of a guiding channel are not included. A comparison with the PIC code WARP [21] is given below to justify our ASTRA simulation and to show their limitations.

\section{A. Astra simulations}

In the following, we simulate a complete laser-driven plasma stage, boosting a $100 \mathrm{MeV}$ electron beam from a conventional linac to $1.0 \mathrm{GeV}$ over a $21 \mathrm{~cm}$ plasma stage. 
We assume typical parameters for an ultrashort electron bunch produced by a conventional accelerator $[22,23]$ of $\Delta \gamma / \gamma=0.1 \%$ energy spread, rms bunch length $\sigma_{z}=1 \mu \mathrm{m}$, and transverse normalized emittance $\epsilon_{n x}=1 \mathrm{mmmrad}$. The charge of these ultrashort bunches is limited by space charge effects. We therefore choose it very low with $Q=0.1 \mathrm{pC}$, which also makes the influence of beam loading effects negligible. Laser parameters are similar to current terawatt class laser systems: $a_{0}=0.8$, $w_{0}=26 \mu \mathrm{m}, z_{R}=2.7 \mathrm{~mm}, \sigma_{z}=13 \mu \mathrm{m}$, and $1.5 \mathrm{~J}$ pulse energy.

The setup of our simulation is shown in Fig. 2. The plasma density profile rises linearly over $10 z_{R}$ in the incoupling section from vacuum to a density of $n_{\text {accel }}=1 \times 10^{17} \mathrm{~cm}^{-3}$, $\lambda_{p}=106 \mu \mathrm{m}$ and stays constant throughout the acceleration section of $13 \mathrm{~cm}$ length and the extraction section of $5 \mathrm{~cm}$ length. The laser follows the paraxial beam evolution to its focus at the beginning of the acceleration section and then stays guided by a density channel until the end of the stage, while in the acceleration section its spot size is constant at $w=w_{0}$. In the extraction section we taper $K$ by linearly increasing $w$ as in Eq. (5) with $g=137 \mathrm{~m}^{-1}$, and $g \beta_{0}=0.2$. Figure 2(a) shows the evolution of the emittance (red line) and the electron beam size (black line) along the complete plasma stage. For comparison, we also show a simulation with sharp plasma-vacuum transition in Fig. 2(b). The plasma density and laser evolution are schematically indicated by the shaded red and gray areas, respectively.

We use $\beta=4 \mathrm{~cm}$ and $\alpha=1.6$ at the beginning of the up ramp to couple into the plasma, which corresponds to a beta function of $\beta_{\mathrm{foc}}=1.1 \mathrm{~cm}$ in the virtual focus. This greatly relaxes the injection beam optics compared to the matched beta function of $0.8 \mathrm{~mm}$ required to couple directly into the acceleration section assuming a sharp transition. Furthermore, positioning tolerances of the laser and injected bunch, stemming from unavoidable fluctuations of the laser and electron beam, are greatly improved by using the plasma up ramp. In Fig. 3, we show the emittance growth at the beginning of the acceleration section for the case of a sharp vacuum-plasma transition (b) and a tapered plasma up ramp (a), assuming positioning jitters of $10 \%$ of the rms electron and laser spot size. For the sharp transition we find an emittance growth of $20 \%$ compared to only $2 \%$ for the plasma up ramp.

During the acceleration, the $100 \mathrm{MeV}$ bunch is boosted to an energy of $E=1.0 \mathrm{GeV}$ and accumulates a correlated energy spread of $\Delta E / E / \sigma_{z}=2.2 \% / \mu \mathrm{m}$. The normalized emittance is literally not changed.

In the extraction section, the divergence of the bunch is reduced by a factor of 4 to $130 \mu \mathrm{rad}$ compared to $550 \mu \mathrm{rad}$ in the untapered case; see Fig. 2. This leads to very small emittance growth in the drift after the plasma of only $\Delta \epsilon_{n x} / \Delta z=0.18 \mathrm{~mm} \mathrm{mrad} / \mathrm{m}$, which is an improvement by more than one order of magnitude compared to $12 \mathrm{~mm} \mathrm{mrad} / \mathrm{m}$ for the sharp plasma-vacuum transition.
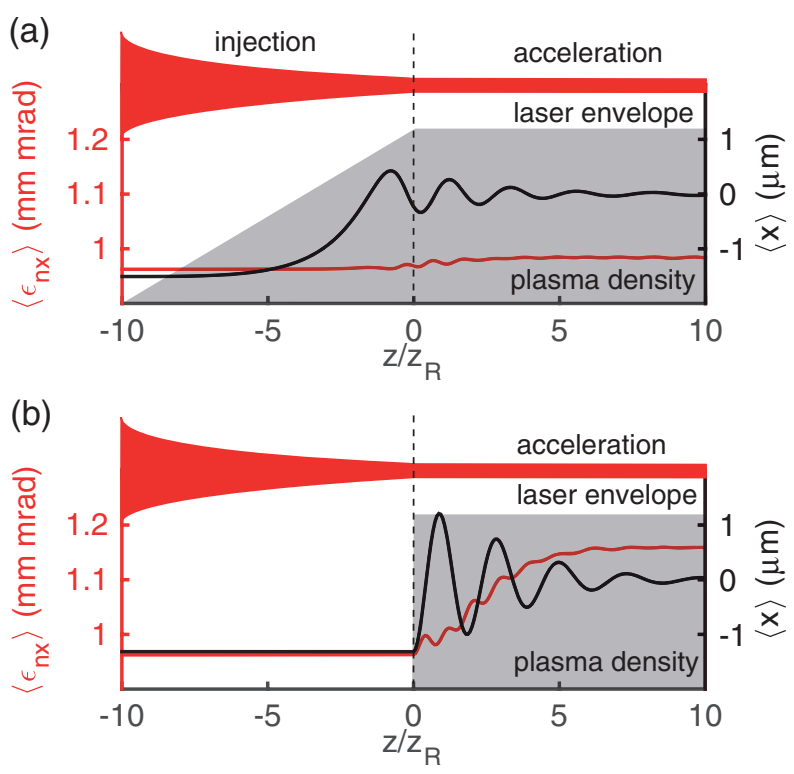

FIG. 3. A proper matching into the acceleration section of the plasma relaxes the tolerances to beam position jitter. The emittance (red line) is averaged over 3000 independent runs, with a laser and bunch position jitter of $10 \% \mathrm{rms}$ of their respective spot size at the focus. Gray and red areas indicate the plasma density profile and laser envelope, respectively. The emittance effectively stays constant when including the matching section (a), while it increases by about $20 \%$ if the laser and electron beam are directly focused to the entrance of the acceleration channel (b). The exemplary trajectory of a bunch initially displaced by $1 \sigma$ of the position jitter is shown in black. The matching section reduces the oscillation amplitude at the laser focus and therefore the emittance growth.

\section{B. Comparison to PIC simulations}

In order to justify the applicability of ASTRA to the case shown above, we run a 2D PIC simulation using WARP in the Lorentz boosted frame $\left(\gamma_{\text {boost }}=10\right)$ of the complete stage as shown in Fig. 2(a). The dimensions of the box were $148 \times 1560 \mu \mathrm{m}$ with $5541 \times 4681$ cells and, because of the computational cost, only one particle per cell. The comparison of ASTRA and WARP is shown in Fig. 4.

In contrast to the ASTRA simulation, where the laser spot size is assumed to evolve according to an analytical function, the PIC simulation self-consistently models the propagation through the guiding channel. Changes in the laser spot size then are achieved by tailoring the channel depth. At the transition from constant spot size in the acceleration section to linearly increasing $w(z)$ in the extraction section, the channel depth is changed slowly so that the laser spot size can adapt to it and stay matched in the channel. Here, this transition region is five Rayleigh lengths long.

The laser evolution in 2D differs from the one in 3D. Therefore, we show in solid black another AsTRA run using the 2D laser model to allow for direct comparison with the 2D PIC simulation. 


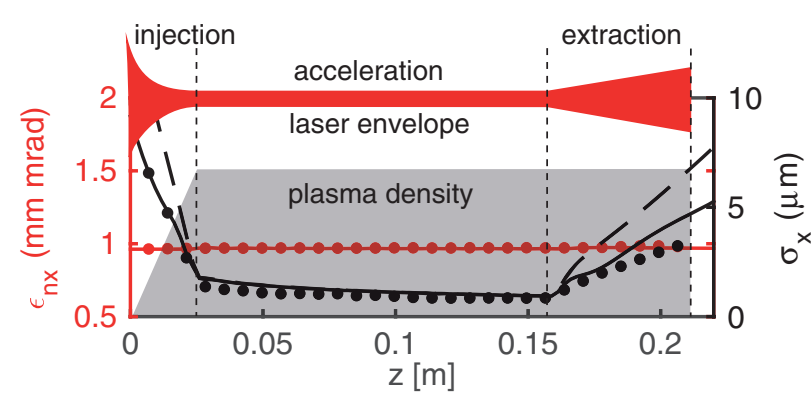

FIG. 4. Comparison of the 3D AstRA run as shown in Fig. 2(a) (dashed line) and a 2D PIC run (dots). The dashed black line shows the beam size evolution according to the 3D wakefield model in ASTRA. For comparison, the solid black line corresponds to an ASTRA simulation employing the same 2D laser model as the PIC simulation.

Effects like wakefield nonlinearities and the influence of the guiding channel lead to a different matched beam size in the PIC run $\left(\sigma_{x}=1.2 \mu \mathrm{m}\right.$ at $\left.z=3.5 \mathrm{~cm}\right)$ than in the ASTRA simulations $\left(\sigma_{x}=1.6 \mu \mathrm{m}\right)$. Also, the beam size evolution in the extraction section is slightly different in the PIC code, as can be seen in Fig. 4. However, since all changes in $K$ are adiabatic, the emittance is conserved in all simulations.

Instead of tailoring the laser evolution, one could also taper the plasma density according to Eq. (6). However, since this ramp is very steep, it might be prone to trigger unwanted down ramp injection. Also, tailoring $n(z)$ is only reasonable without a guiding channel. Since the tapered density drops quickly by orders of magnitude, the use of a plasma density channel is not only challenging from a technological point of view but leads to strong deformation of the wakefield at low densities.

(a)
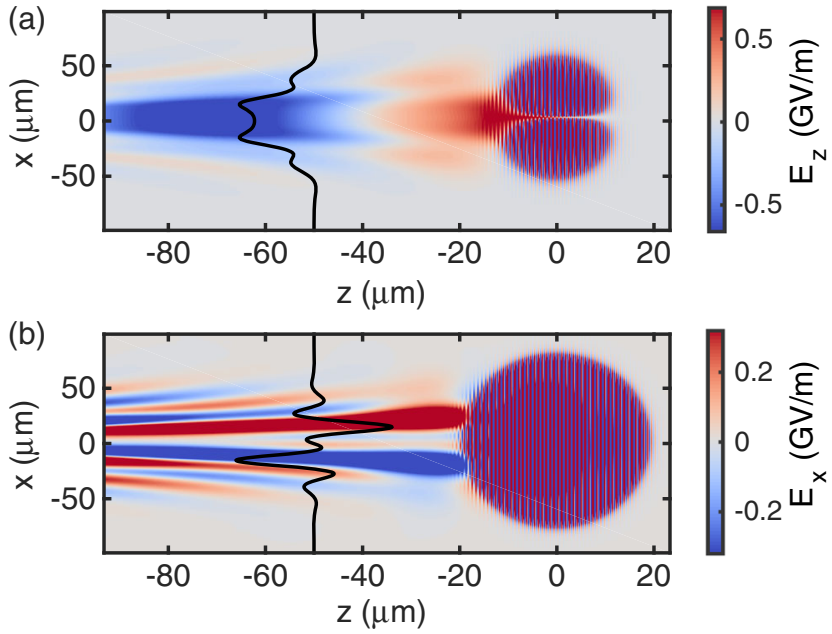

FIG. 5. Wakefield in a low-density guiding channel $\left(n=1 \times 10^{15} \mathrm{~cm}^{-3}\right)$. (a) and (b) show the longitudinal and transverse electric field, respectively. The lineouts of the fields $50 \mu \mathrm{m}$ behind the laser show a dip in $E_{z}$ on axis and a change of sign in $E_{x}$, which is defocusing to electrons.
In Fig. 5, we use WARP in 2D to simulate a wakefield in a parabolic guiding channel of on-axis density $1 \times 10^{15} \mathrm{~cm}^{-3}$, driven by a laser of matched spot size $w_{0}=26 \mu \mathrm{m}$ and $25 \mathrm{fs}$ FWHM pulse length. This density would be present after $1 \mathrm{~cm}$ in the $5 \mathrm{~cm}$ long plasma density down ramp. Box dimensions are $117 \times 260 \mu \mathrm{m}$ divided into $8741 \times 780$ cells with 16 particles per cell. Off axis, the wakefield is stronger; therefore, a dip appears in $E_{z}$ on axis. $E_{x}$ is defocusing for electrons on axis and focusing off axis. In such a field, the beam electrons will be defocused and form a hollow cylindrical bunch in the off-axis focusing regions, leading to strong emittance growth. In some cases featuring driver lasers of large spot size and consequently long Rayleigh length, it is possible to design a density tapered extraction section [24]. Yet, especially for staged acceleration concepts, efficiency is a crucial issue, which is why they rely on laser guiding. Density tapering is not an option then.

\section{DISCUSSION}

For the simulations shown here, the extraction length and $g$ are chosen to show the general capability of the concept and need to be subject to optimization regarding specific future experiments, as they influence the divergence after the plasma. Also, we can choose a length of the plasma up ramp shorter than the down ramp, since the injected electron energy is much smaller before acceleration. As the emittance stays constant in the up ramp, adiabaticity is still fulfilled. For larger energies and larger energy spread beams, the up ramp would be longer for two reasons: first, in order to maintain adiabaticity at higher energies and, second, to maximize the beta function and minimize divergence in the drift before the plasma section to prevent chromatic emittance growth. Eventually, the up ramp profile would follow the design of the down ramp.

Furthermore, the bunch charge is very low. The influence of beam loading for beams of higher charge, as one would expect from a laser-driven source, needs further investigation. The wake driven by the beam itself could hamper the decay of the focusing strength especially in a matching section where only the laser spot size is tailored. On the other hand, beam loading could prevent unwanted injection in a density down ramp.

Recently, it was proposed to introduce a laser-driven plasma lens behind the target, separated by a short drift [25]. Depending on the specific setup, also the combination of a plasma lens and an adiabatic extraction section could be beneficial. However, since the plasma lens relies on the remaining laser intensity of the diffracting laser, a combination with a laser tapered extraction section is likely not efficient. Further studies on the combination with a density down ramp are needed.

In conclusion, we show that by properly tapering the plasma entrance and exit sections we drastically simplify the required beam optics to match into the plasma, reduce 
sensitivity to positioning jitters, and enable conservation of the excellent beam emittance from the plasma to the subsequent drift section. We find that tapering the laser intensity is favorable to tapering the density, because of strong distortions of the wake in low-density guiding channels and the risk of down ramp injection. Compared to untapered scenarios, the highly reduced divergence is a prerequisite for subsequent beam optics. This technique is essential for a plasma booster or staged accelerators in future high-energy $[3,4]$ and compact light source applications $[26,27]$.

Although we focus on external injection schemes, our discussion naturally applies to triggered injection schemes in the linear or quasilinear regime, such as colliding pulse injection [28]. Our concept works also for veryhigh-energy spread beams; yet, a general reduction of beam energy spread is highly favorable considering further beam transport and applications.

\section{ACKNOWLEDGMENTS}

We gratefully acknowledge the computing time provided on the supercomputer JUROPA under Project No. HHH20. I. D. acknowledges support by the IMPRS UFAST. We thank N. Delbos (CFEL/UHH), M. Kirchen (CFEL/UHH), and $\mathrm{C}$. Werle (CFEL/UHH) for stimulating discussions and useful suggestions.

[1] T. Tajima and J. M. Dawson, Phys. Rev. Lett. 43, 267 (1979).

[2] E. Esarey, C. B. Schroeder, and W. P. Leemans, Rev. Mod. Phys. 81, 1229 (2009).

[3] W. P. Leemans and E. Esarey, Phys. Today 62, No. 3, 44 (2009).

[4] C. B. Schroeder, E. Esarey, C. G. R. Geddes, C. Benedetti, and W. P. Leemans, Phys. Rev. ST Accel. Beams 13, 101301 (2010).

[5] W. P. Leemans, A. J. Gonsalves, H.-S. Mao, K. Nakamura, C. Benedetti, C. B. Schroeder, C. Tóth, J. Daniels, D. E. Mittelberger, S. S. Bulanov, J.-L. Vay, C. G. R. Geddes, and E. Esarey, Phys. Rev. Lett. 113, 245002 (2014).

[6] H. T. Kim, K. H. Pae, H. J. Cha, I. J. Kim, T. J. Yu, J. H. Sung, S. K. Lee, T. M. Jeong, and J. Lee, Phys. Rev. Lett. 111, 165002 (2013).

[7] X. Wang, R. Zgadzaj, N. Fazel, Z. Li, S. A. Yi, W. Henderson, Y.-Y. Chang, R. Korzekwa, H.-E. Tsai, C. Pai, H. Quevedo, G. Dyer, E. Gaul, M. Martinez, A. C. Bernstein, T. Borger, M. Spinks, M. Donovan, V. Khudik, G. Shvets, T. Ditmire, and M. C. Downer, Nat. Commun. 4, 1988 (2013).

[8] P. Antici, A. Bacci, C. Benedetti, E. Chiadroni, M. Ferrario, A. R. Rossi, L. Lancia, M. Migliorati, A. Mostacci, L. Palumbo, and L. Serafini, J. Appl. Phys. 112, 044902 (2012).

[9] M. Migliorati, A. Bacci, C. Benedetti, E. Chiadroni, M. Ferrario, A. Mostacci, L. Palumbo, A. R. Rossi, L. Serafini, and P. Antici, Phys. Rev. ST Accel. Beams 16, 011302 (2013).

[10] K. Floettmann, Phys. Rev. ST Accel. Beams 6, 034202 (2003).

[11] A. Cianchi, M. Anania, M. Bellaveglia, M. Castellano, E. Chiadroni, M. Ferrario, G. Gatti, B. Marchetti, A. Mostacci, R. Pompili, C. Ronsivalle, A. Rossi, and L. Serafini, Nucl. Instrum. Methods Phys. Res., Sect. A 720, 153 (2013).

[12] G. R. Plateau, C. G. R. Geddes, D. B. Thorn, M. Chen, C. Benedetti, E. Esarey, A. J. Gonsalves, N. H. Matlis, K. Nakamura, C. B. Schroeder, S. Shiraishi, T. Sokollik, J. van Tilborg, C. Toth, S. Trotsenko, T. S. Kim, M. Battaglia, T. Stöhlker, and W. P. Leemans, Phys. Rev. Lett. 109, 064802 (2012).

[13] C. M. S. Sears, A. Buck, K. Schmid, J. Mikhailova, F. Krausz, and L. Veisz, Phys. Rev. ST Accel. Beams 13, 092803 (2010).

[14] R. Weingartner, S. Raith, A. Popp, S. Chou, J. Wenz, K. Khrennikov, M. Heigoldt, A. R. Maier, N. Kajumba, M. Fuchs, B. Zeitler, F. Krausz, S. Karsch, and F. Grüner, Phys. Rev. ST Accel. Beams 15, 111302 (2012).

[15] T. Mehrling, J. Grebenyuk, F. S. Tsung, K. Floettmann, and J. Osterhoff, Phys. Rev. ST Accel. Beams 15, 111303 (2012).

[16] R. Assmann and K. Yokoya, Nucl. Instrum. Methods Phys. Res., Sect. A 410, 544 (1998).

[17] L. M. Gorbunov and V. I. Kirsanov, Sov. Phys. JETP 93, 509 (1987).

[18] A. J. Gonsalves, T. P. Rowlands-Rees, B. H. P. Broks, J. J. A. M. van der Mullen, and S. M. Hooker, Phys. Rev. Lett. 98, 025002 (2007).

[19] K. Floettmann, Phys. Rev. ST Accel. Beams 17, 054402 (2014).

[20] K. Floettmann, http://www.desy.de/ mpyflo/.

[21] A. Friedman, R. H. Cohen, D. P. Grote, S. M. Lund, W. M. Sharp, J.-L. Vay, I. Haber, and R. A. Kishek, IEEE Trans. Plasma Sci. 42, 1321 (2014).

[22] A. Bacci and A. R. Rossi, Nucl. Instrum. Methods Phys. Res., Sect. A 740, 42 (2014).

[23] M. J. Nasse, E. Huttel, S. Marsching, A.-S. Müller, S. Naknaimueang, R. Rossmanith, R. Ruprecht, M. Schreck, M. Schuh, M. Schwarz, P. Wesolowski, R. W. Assmann, M. Felber, K. Floettmann, M. Hoffmann, H. Schlarb, H.-H. Braun, R. Ganter, and L. Stingelin, in Proceedings of the 4th International Particle Accelerator Conference, IPAC2013, Shanghai, China, 2013 (JACoW, Shanghai, China, 2013), WEPWA010.

[24] X. Xu, Y. Wu, C. Zhang, F. Li, Y. Wan, J. F. Hua, C.-H. Pai, W. Lu, P. Yu, W. An, W. B. Mori, C. Joshi, and M. J. Hogan, arXiv:1411.4386.

[25] R. Lehe, C. Thaury, E. Guillaume, A. Lifschitz, and V. Malka, Phys. Rev. ST Accel. Beams 17, 121301 (2014).

[26] A. R. Maier, A. Meseck, S. Reiche, C. B. Schroeder, T. Seggebrock, and F. Grüner, Phys. Rev. X 2, 031019 (2012).

[27] Z. Huang, Y. Ding, and C. B. Schroeder, Phys. Rev. Lett. 109, 204801 (2012).

[28] J. Faure, C. Rechatin, A. Norlin, A. Lifschitz, Y. Glinec, and V. Malka, Nature (London) 444, 737 (2006). 